\title{
Statistical Synthesis of Robust Signal Detection Algorithms under Conditions of Aprioristic Uncertainty
}

\section{G. Prokopenko}

National Aviation University, Kosmonavta Komarova ave. 1, 03680, Kyiv, Ukraine

Email:prokop-igor@yandex.ua

Abstract: The paper deals with the problem of synthesis of a robust detection algorithm for a harmonic signal on the background of correlated noise and impulse noise. The problem is solved using the empirical Bayes approach and the Tukey model of "pollution". The efficiency of the algorithm is investigated by the MonteCarlo method.

Keywords: Signal processing, aprioristic uncertainty, algorithm, robustness, radar signal, correlated noise, pulse interference.

\section{Introduction}

The problem of synthesis of robust signal processing algorithms arises in many areas of technology - communication, navigation, radar systems and processing of biomedical and acoustic signals [1-4]. When these tasks are solved, known statistical models of probability distributions mixtures of signal and noise are used. In real conditions, the synthesized parametric algorithms for signals detection and estimation of their parameters do not retain their qualities (the probability of errors of the first and second kind, the accuracy of estimates) with a deviation of the actual distributions from those adopted at the synthesis stage [1]. In order to provide robustness of the signal processing algorithms, it is possible to use a not fully certain statistical model of signals and interference distribution.

In the general case the aprioristic uncertainty can be described by the Tukey model of interference probability density distribution [2].

$$
f_{0}(x)=(1-p) f_{n}(x, \bar{\theta})+p h(x),
$$

where $f_{n}(x, \bar{\theta})$ is a known Probability Density Function (PDF) according to the minimax approach, $h(x)$ is, as a rule, an exponential or Laplace, $p$ is the probability 
of the interference action named "neighborhood pollution" or "Tukey neighborhood", $\bar{\theta}$ is a vector of the parameters. In Fig. 1 one can see "Tukey neighborhood" of normal distribution with Laplace "pollution".

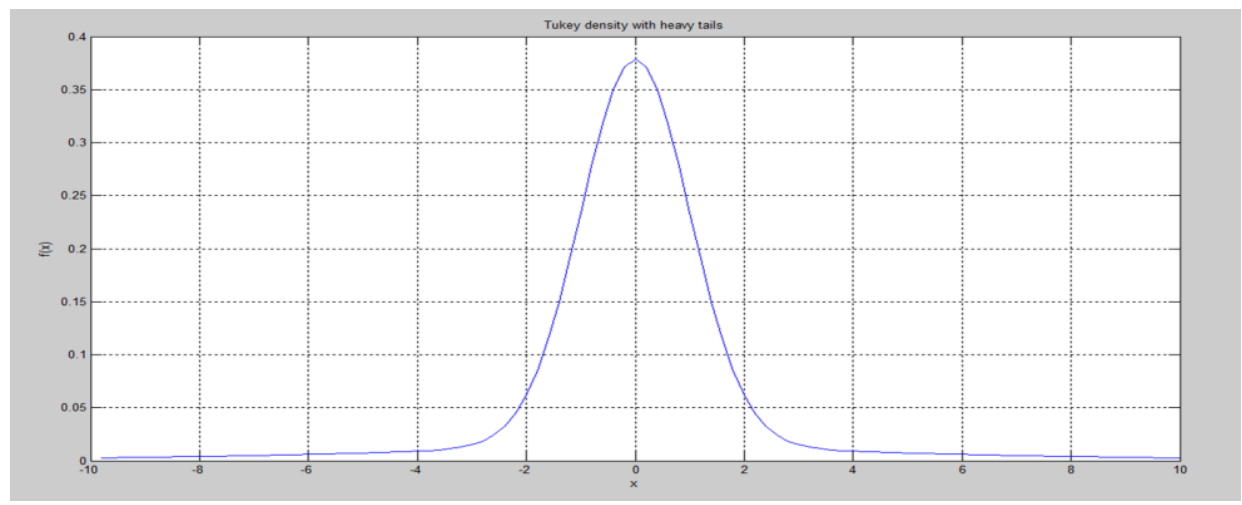

Fig. 1. Tukey model of interference probability density distribution with Laplace "pollution"

We introduce the parameter $b$, which will characterize the signal/noise ratio. Signal and noise mixture, distributed similarly is

$$
f_{1}(x)=(1-p) f_{s}(x, b, \bar{\theta})+p h(x),
$$

where $f_{s}(x, b, \bar{\theta})$ is the known PDF of the signal.

Signal detection task statement. Let us have a sample $\bar{X}=\left(x_{1}, \ldots, x_{n}\right)$ relatively to which two statistical hypotheses are checked. These hypotheses are:

$H_{0}: f(\bar{X})=f_{0}(\bar{X}, \bar{\theta})$ that means: the sample relates to an interference area.

$H_{1}: f(\bar{X})=f_{1}(\bar{X}, \bar{\theta})$, that is, the sample relates to a signal area.

The decision rule in this case is given according to Neyman-Pearson lemma by the likelihood ratio

$$
l\left(x_{1}, \ldots, x_{n}\right)=\frac{f_{1}(\bar{X}, \bar{\theta})}{f_{0}(\bar{X}, \bar{\theta})}>V(\alpha),
$$

where $V(\alpha)$ is a threshold of decision making, $\alpha$ is the probability of false alarm.

Taking into account (1) and (2), the likelihood ratio obtains the following form

$$
l\left(x_{1}, \ldots, x_{n}\right)=\prod_{i=1}^{n} \frac{(1-p) f_{s}\left(x_{i}, b, \bar{\theta}\right)+p h\left(x_{i}\right)}{(1-p) f_{n}\left(x_{i}, \bar{\theta}\right)+p h\left(x_{i}\right)}>V(\alpha) .
$$

We can transform this equation into

$$
l\left(x_{1}, \ldots, x_{n}\right)=\prod_{i=1}^{n} \frac{\lambda\left(x_{i}, \bar{\theta}\right)+M\left(x_{i}\right)}{1+M\left(x_{i}\right)}>V(\alpha),
$$

where $\lambda\left(x_{i}, \bar{\theta}\right)=\frac{f_{s}\left(x_{i}, b, \bar{\theta}\right)}{f_{n}\left(x_{i}, \bar{\theta}\right)}$ is a parametrical likelihood ratio, and $M\left(x_{i}\right)$, $M\left(x_{i}\right)=\frac{p h\left(x_{i}\right)}{(1-p) f_{n}\left(x_{i}, \bar{\theta}\right)}$ is a stabilizing term. 
It takes large values for interference emissions. If $x_{i}$ belongs to noise, $M$ takes a small value.

In this paper we consider the synthesis of a robust harmonic signal detection algorithm in mixture of correlated noise and impulse interference.

\section{Detection of a harmonic signal on the background of correlated noise and pulse interference}

The task of a harmonic signal detection on the background of correlated interference is well known. This task is characteristic for radar detection of moving objects on the background of unwanted signals, reflected by the land or by meteorological objects.

Let us consider a task of harmonic signal detection in mixture with a correlated noise and a pulse clatter.

$$
x_{i}=b \cos \left(\omega t_{i}+\varphi\right)+\eta_{i}+I_{i}, i=1, \ldots, n,
$$

where $\eta_{i}$ is a correlated noise sequence, $I_{i}$ is the pulse clatter, $\omega$ is a signal frequency, $\varphi$ is an initial phase, $b$ is a signal amplitude.

The wide class of random processes supposes approximation by an autoregressive process. The sequence of samples of this process can be presented by the following model

$$
\eta_{i}=\sum_{j=1}^{k} a_{j} \eta_{i-j}+\xi_{i},
$$

where $a_{j}, j=1, \ldots, k$, are autoregressive coefficients, $\xi_{i}$ are independent samples of generating a normal random process. In this model the parameter vector is $\bar{\theta}=\left[a_{1}, \ldots, a_{k}\right]$.

This model presents a process at the output of a linear system when at the input of this system a normal uncorrelated random process acts. This is the socalled autoregressive $k$-order process or $k$-order Markov process. The multivariate PDF of such a process can be written as

$$
f\left(x_{1}, \ldots, x_{n}, \bar{\theta}\right)=\frac{1}{\left(\sqrt{2 \pi \sigma^{2}}\right)^{n}} \exp \left\{-\frac{x_{1}^{2}+\sum_{i=2}^{n}\left(x_{i}-\sum_{j=1}^{\min \{i-1, k\}} a_{j} x_{i-j}\right)^{2}}{2 \sigma^{2}}\right\},
$$

where $\sigma^{2}$ is variance of the generating sequence $\xi_{i}$.

The analytical equation of a multivariate density function (6) allows carrying out the synthesis of optimal decision rules with a simple structure. Note that the sequence of the generating process $\xi_{i}, i=1, \ldots, n$, can be non-Gaussian. In this case the probability distribution of the autoregressive sequence is non-Gaussian too. It is possible to use this Markov model to solve the task of a moving target detection on the background of correlated clatters. 
The pulse clatter model is given like a sequence of random numbers with PDF

$$
f_{I}(x)=(1-p) \delta(x)+p \frac{g}{2} \exp (-g|x|),
$$

where $p$ is the probability of the pulse clatter action, $g$ is a scale parameter of Laplace distribution. The pulse clatter with this probability distribution is the most harmful.

The conditional probability distribution of mixture (5) with respect to (6) and (7) has the following form:

$$
\begin{gathered}
f\left(x_{i} \mid x_{i-1}, \ldots x_{i-k}, \bar{\theta}, b\right)=(1-p) \frac{1}{\sqrt{2 \pi} \sigma} \exp \left\{-\frac{\left(x_{i}-\sum_{j=1}^{k} a_{j} x_{i-j}-b\left(S_{i}-\sum_{j=1}^{k} a_{j} S_{i-j}\right)\right)^{2}}{2 \sigma^{2}}\right\}+ \\
+\frac{p g}{2} \exp \left(-g \mid x_{i}\right),
\end{gathered}
$$

where $S_{i}=b \cos \left(\omega t_{i}+\phi\right), i=1, \ldots, n$, are signal values. The parameter vector is $\bar{\theta}=\left[a_{1}, \ldots, a_{k}, g, \varphi, \sigma^{2}\right]$.

Using equations (3), (4) and (8), one can obtain a robust detection algorithm

$$
l\left(x_{1}, \ldots, x_{n}\right)=\prod_{i=1}^{n} \frac{\lambda\left(x_{i}, \bar{\theta}\right)+M\left(x_{i}\right)}{1+M\left(x_{i}\right)}=\prod_{i=1}^{n} l\left(x_{i}, \bar{\theta}\right)>V(p, \alpha),
$$

where

$$
\begin{gathered}
l\left(x_{i}, \bar{\theta}\right)=\frac{f\left(x_{i} \mid x_{i-1}, \ldots, x_{i-k}, \overline{\theta, b}\right)}{f\left(x_{i} \mid x_{i-1}, \ldots x_{i-k}, \bar{\theta}, b=0\right)}=\frac{\exp \left(\left(x_{i}-\sum_{j=1}^{k} a_{j} x_{i-j}\right)\left(S_{i}-\sum_{j=1}^{k} a_{j} S_{i-j}\right) / 2 \sigma^{2}\right)+M\left(x_{i}\right)}{1+M\left(x_{i}\right)}, \\
M\left(x_{i}\right)=\frac{\sqrt{2 \pi} \sigma p g \exp \left(-g\left|x_{i}\right|\right)}{2(1-p) \exp \left(-\left(x_{i}-\sum_{j=1}^{k} a_{j} x_{i-j}\right)^{2} / 2 \sigma^{2}\right)} .
\end{gathered}
$$

We consider the situation when the initial phase of the signal is unknown. Given that the type of the signal $S_{i}=b \cos \left(\omega t_{i}+\phi\right), i=1, \ldots, n, l\left(x_{i}, \bar{\theta}\right)$ can be expressed as

$$
l\left(x_{i}, \bar{\theta}\right)=\frac{\exp \left(\left(\mathrm{A}\left(x_{i}, \omega\right) \cos (\varphi)+\mathrm{C}\left(x_{i}, \omega\right) \sin (\varphi)\right) / 2 \sigma^{2}\right)+M\left(x_{i}\right)}{1+M\left(x_{i}\right)},
$$

where

$$
\begin{aligned}
& A\left(x_{i}, \omega\right)=b\left(x_{i}-\sum_{j=1}^{k} a_{j} x_{i-j}\right)\left(\cos \left(\omega t_{i}\right)-\sum_{j=1}^{k} a_{j} \cos \left(\omega t_{i-j}\right)\right), \\
& C\left(x_{i}, \omega\right)=b\left(x_{i}-\sum_{j=1}^{k} a_{j} x_{i-j}\right)\left(\sin \left(\omega t_{i}\right)-\sum_{j=1}^{k} a_{j} \sin \left(\omega t_{i-j}\right)\right) .
\end{aligned}
$$

This allows us to introduce $l\left(x_{i}, \bar{\theta}\right)$ in the following form:

$$
l\left(x_{i}, \bar{\theta}\right)=\frac{\exp \left(\left(R\left(x_{i}, \omega\right) \cos \left(G\left(x_{i}, \omega\right)+\varphi\right)\right) / 2 \sigma^{2}\right)+M\left(x_{i}\right)}{1+M\left(x_{i}\right)},
$$


where $R\left(x_{i}, \omega\right)=\sqrt{A\left(x_{i}, \omega\right)^{2}+C\left(x_{i}, \omega\right)^{2}}, G\left(x_{i}, \omega\right)=\operatorname{arctg}\left[C\left(x_{i}, \omega\right) / A\left(x_{i}, \omega\right)\right]$.

To obtain a phase invariant algorithm, we should integrate this statistics over $\varphi$ :

$$
\begin{gathered}
\lambda\left(x_{i}\right)=\int_{0}^{2 \pi} \frac{\exp \left(b R\left(x_{i}, \omega\right) / \sigma^{2}\right) \cos (\varphi-G(\omega))+M\left(x_{i}\right)}{1+M\left(x_{i}\right)} d \varphi= \\
=\frac{I_{0}\left(b R\left(x_{i}, \omega\right) / \sigma^{2}\right)+M\left(x_{i}\right)}{1+M\left(x_{i}\right)},
\end{gathered}
$$

where $I_{0}(z)$ is a modified Bessel function of zero order.

Using the approximation of Bessel function in the area $z \in[0,10]$

$$
I_{0}(z) \approx \frac{\exp (z / 1.15)}{\sqrt{2 \pi}}
$$

we can transform the decision rule (9) into the following form

$$
l\left(x_{1}, \ldots, x_{n}, \bar{\theta}\right)=\prod_{i=1}^{n} \frac{\exp \left(R\left(x_{i}, \omega\right) / 1.15\right) / \sqrt{2 \pi}+M\left(x_{i}\right)}{1+M\left(x_{i}\right)}>V(p, \alpha) .
$$

In Fig. 2 the dependency of the logarithm of one factor of the product (9) on both $x$ and the scale parameter of a pulse clatter $g$ is shown (the variance of noise $\left.\sigma^{2}=1\right)$.

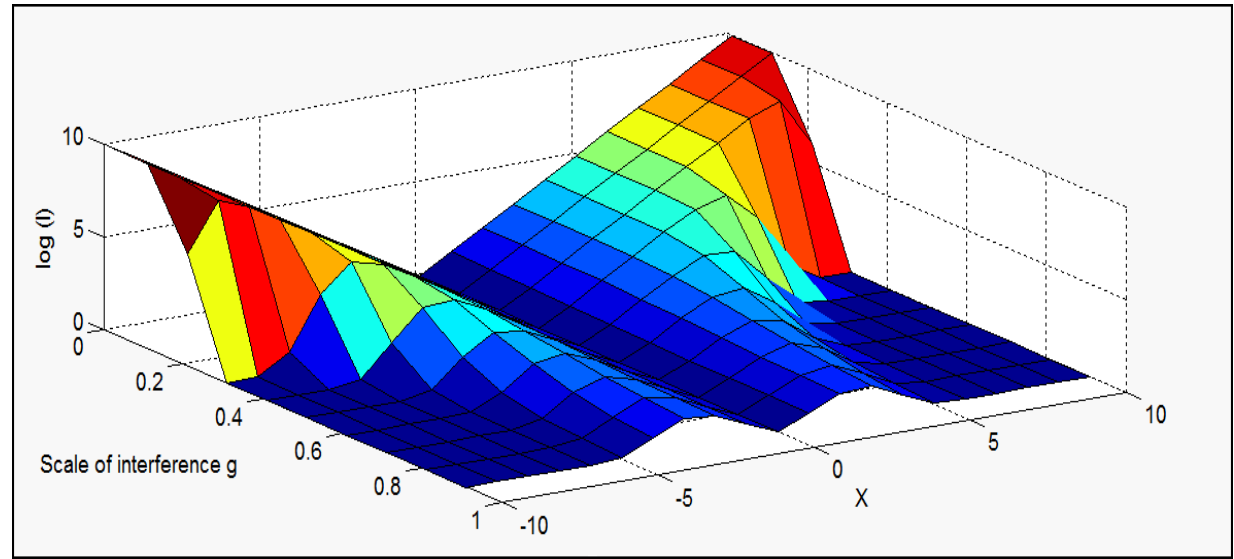

Fig. 2. Dependency of the logarithm of one factor of the product (9) on both $x$ and the scale parameter of the pulse clatter $g$. The variance of noise $\sigma^{2}=1$

One can see that the small clatter scale characteristic is V-shaped. The increase of the pulse clatter scale parameter $\mathrm{g}$ transforms this characteristic in the area of big values. The high values are reduced.

Efficiency analysis. The efficiency of the developed algorithms was investigated by Monte-Carlo methods. According to these methods samples of the signal and noise mixture are generated by the following algorithms:

- correlated noise sequence

$$
\eta_{i+1}=\sum_{j=1}^{k} a_{j} \eta_{i-j}+\xi_{i}, i=1, \ldots, n,
$$


$a_{j}, j=1, \ldots, k$, are autoregressive coefficients, $\xi_{i}, i=1, \ldots, n$, is a sequence of normal random numbers;

- mixture of signal, noise and pulse interference

$$
x_{i}=b \cos \left(\omega t_{i}+\varphi\right)+\eta_{i}+I_{i}, i=1, \ldots, n .
$$

In Fig. 2 one can see examples of a harmonic signal, correlated noise and pulse interference mixture, generated by modelling.

The signal processing algorithm $l\left(x_{1}, \ldots, x_{n}\right)$ was modeled according to Equation (10). We repeated this experiment $T$ times and calculated the probability of correct decisions like a ratio of the correct decisions to the number of experiments $T$.

In Fig. 3 the detection characteristics of the robust algorithm (10) and the known MTI algorithm are shown. The correlation coefficient of noise $r=0.94$. We consider four situations: $p=0 ; p=0.03 ; p=0.06 ; p=0.09$.

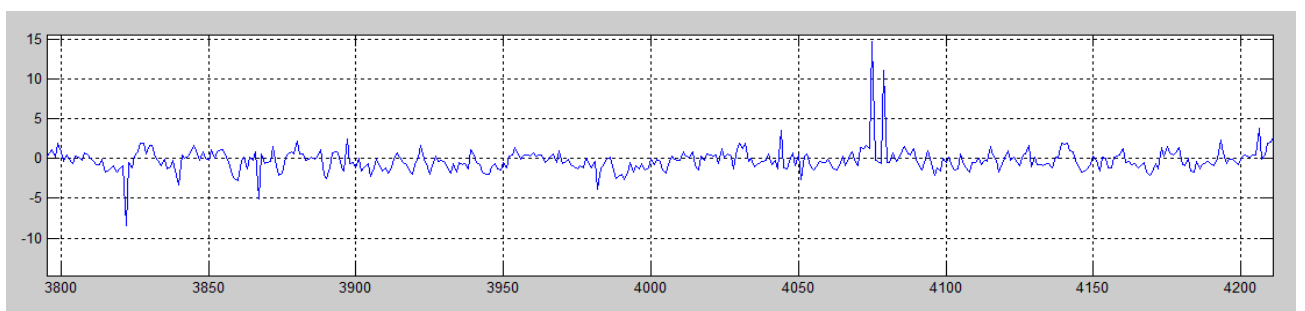

(a)

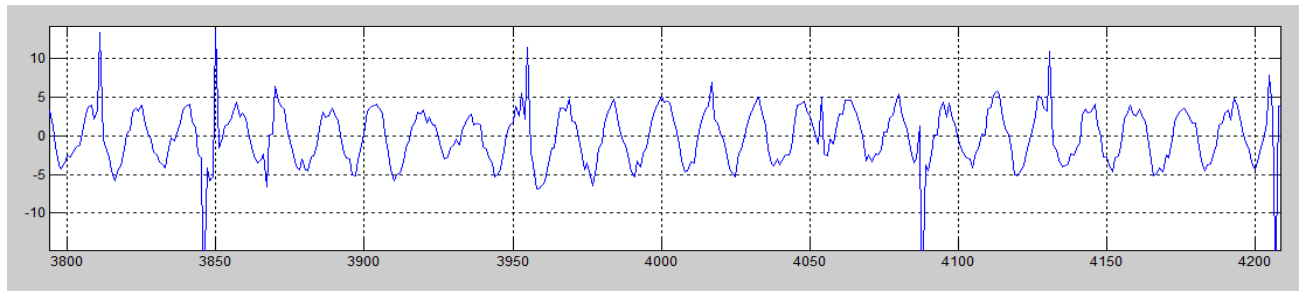

(b)

Fig. 3. Mixture of the correlated noise and pulse interference (a) and a harmonic signal (b) $\left(p=0.04, b=5, \sigma=1, a_{1}=0.6\right)$

The MTI algorithm, investigated in this work is

$$
l\left(x_{1}, \ldots, x_{n}\right)=\sum_{i=2}^{n}\left(x_{i-1}-x_{i}\right)^{2}>V_{d}(\alpha) .
$$

One can see that both robust (the green line) and MTI (11) (the blue line) algorithms are equal, when the pulse interference is absent $(p=0)$. If the probability of the pulse increases $(p=0.03, p=0.06$ and $p=0.09)$, the robust algorithm (10) is more efficient.

Let us consider the situation with aprioristic uncertain autoregressive coefficients of a correlated noise model.

In this case we can use the empirical Bayesian approach, according to which it is necessary to obtain the estimation of these coefficients and substitute them in the likelihood ratio (10). 


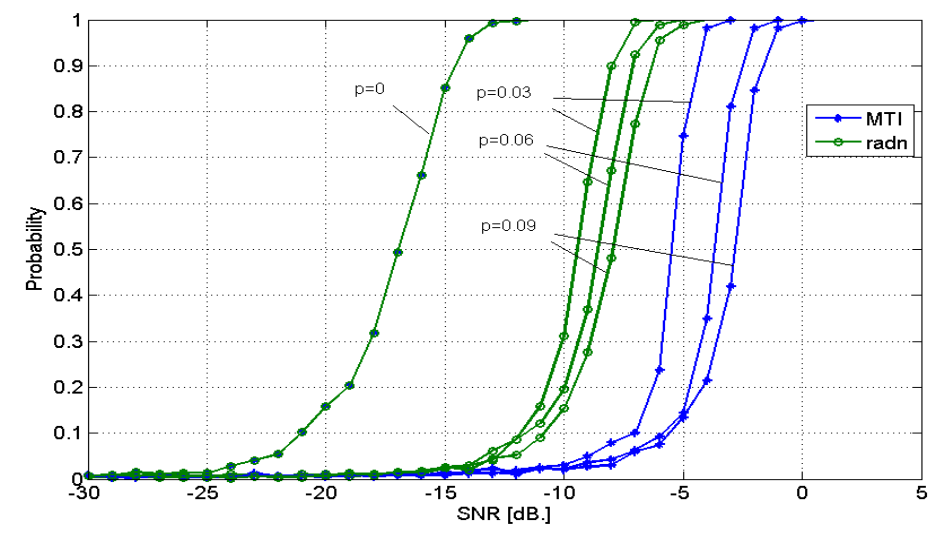

Fig. 4. Detection characteristics of MTI and robust algorithms. Sample size $N=16$, probability of false alarm $\alpha=0.005$, noise correlation coefficient $r=0.94$. The probabilities of the pulse interference:

$$
p=0 ; p=0.03 ; p=0.06 ; p=0.09
$$

To obtain the maximum likelihood estimations of the autoregressive coefficients, we should organize a training sample $y_{1}, \ldots ., y_{m}$ that contains mixture of correlated noise and interference without a signal.

The likelihood function has the following form:

$$
\begin{gathered}
f\left(y_{1}, \ldots, y_{m}, \bar{\theta}\right)=\frac{1}{\left(\sqrt{2 \pi \sigma^{2}}\right)^{m}} \exp \left(-\frac{y_{1}^{2}}{2 \sigma^{2}}\right) \times \\
\times \prod_{i=2}^{m}(1-p) \exp \left\{-\frac{\left(y_{i}-\sum_{j=1}^{\min \{i-1, k\}} a_{j} y_{i-j}\right)^{2}}{2 \sigma^{2}}\right\}+p g \exp \left(-g\left|y_{i}\right|\right) .
\end{gathered}
$$

The likelihood equations can be written as

$$
\begin{gathered}
\frac{\partial f\left(y_{1}, . ., y_{m}, a_{1}, . . a_{k}\right)}{\partial a_{j}}= \\
\sum_{i=2}^{n} \frac{\partial}{\partial a_{j}} \ln \left[(1-p) \exp \left(-\frac{\left(y_{i}-\sum_{j=1}^{\min \{i-1, k\}} a_{j} y_{i-j}\right)^{2}}{2 \sigma^{2}}\right)+p g \exp \left(-g\left|y_{i}\right|\right)\right]=0, j=1, \ldots, k .
\end{gathered}
$$

After some analytical transformations, we obtain the following:

(13) $\sum_{i=1}^{n}\left(\left(y_{i}-\sum_{j=1}^{\min \{i-1, k\}} a_{j} y_{i-j}\right) y_{i-l} /\left(1+\frac{p g \sigma \sqrt{2 \pi}}{1-p} \exp \left(\frac{\left(y_{i}-\sum_{j=1}^{\min \{i-1, k\}} a_{j} y_{i-j}\right)^{2}}{2 \sigma^{2}}-g\left|y_{i}\right|\right)\right)=0\right.$,

$$
l=1, \ldots, k .
$$


Solution of equations (13) can be obtained by Newton-Raphson numerical method and it gives us the robust estimations of the autoregressive coefficients $\theta^{*}=\left(a_{1}^{*}, \ldots, a_{k}^{*}\right)$ that are used for detection of the signal by the algorithm (10).

We analyzed the efficiency of the algorithms for the first order autoregressive process $\left(a_{1}=r\right)$ correlation coefficient estimation. Two algorithms were used robust and non robust.

In Fig. 5 are shown characteristics of the robust estimator of the correlation coefficient obtained according to (13) and characteristics of the non robust algorithm

$$
a_{1}^{*}=\frac{\sum_{i=2}^{n} y_{i} y_{i-1}}{\sum_{i=2}^{n} y_{i}^{2}} \text {. }
$$

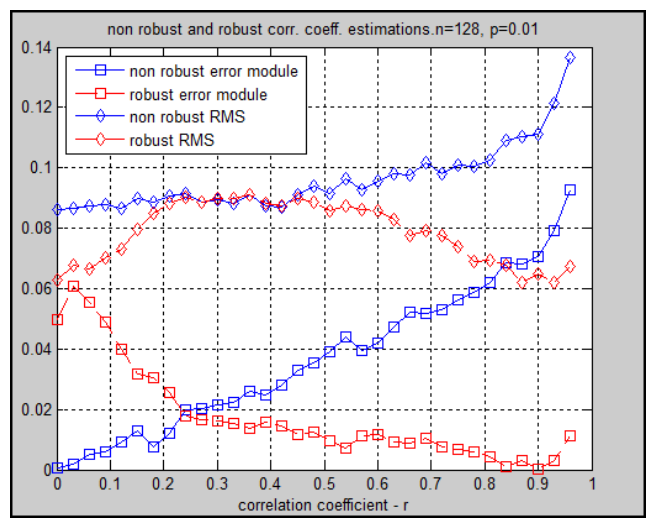

(a)

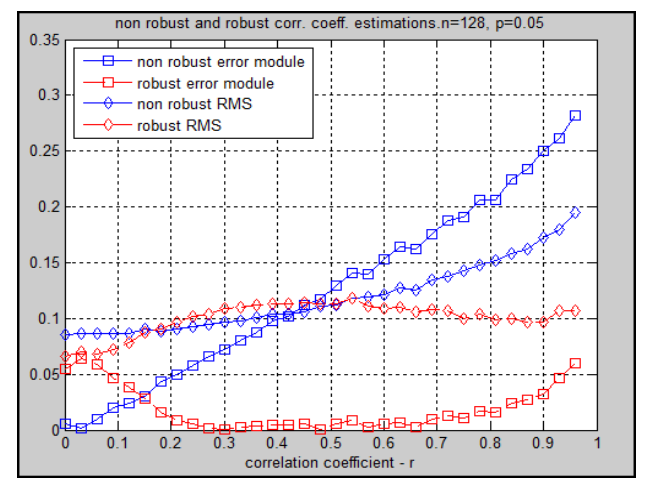

(c)

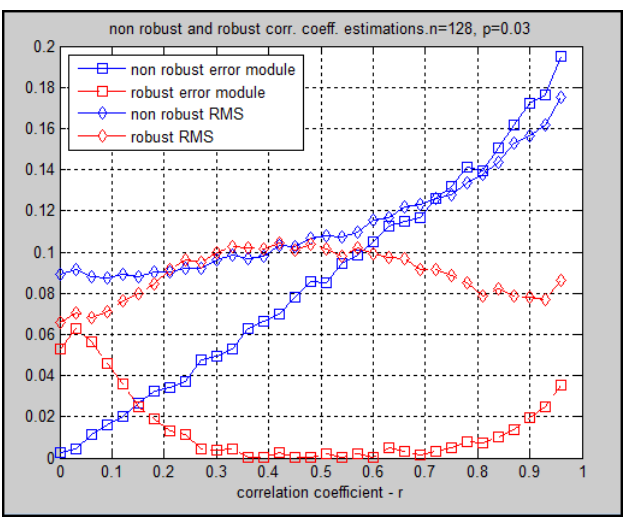

(b)

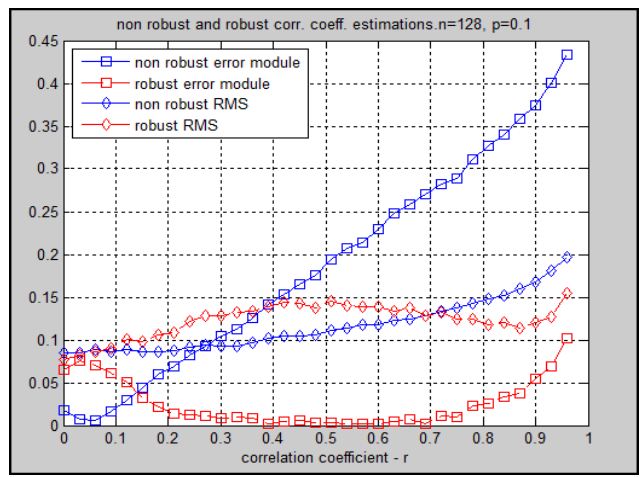

(d)

Fig. 5. Efficiency characteristics of robust and non robust algorithms of noise correlation coefficient estimation in mixture with the pulse interference: (a) $p=0.01$; (b) $p=0.03$; (c) $p=0.05$; (d) $p=0.1$

Every figure contains dependencies of both error modulus and root mean square vs a sample correlation coefficient. 
For every algorithm two characteristics are calculated: modulus of the estimation error vs a correlation coefficient and root mean square vs a correlation coefficient. These characteristics are obtained by Monte-Carlo method for size of the training sample $n=128$ and for different values of the pulse interference probability $(p=0.01,0.03,0.05,0.1)$. If the probability of the pulse interference action is more than 0.01 and the correlation coefficient of noise is more than 0.15 , the robust estimator substantially prevails the non robust one. The advantage increases if the correlation coefficient tends to one.

To estimate the losses of the adaptive to noise correlation coefficient detector, two detection characteristics were calculated (Fig. 6).

One of them belongs to the adaptive detection algorithm used in the estimation of the noise correlation coefficient, solving Equation (13). The sample size $n$ is equal to 16. The second algorithm uses an apriori known correlation coefficient $(r=0.9)$, variance of the correlated noise $\sigma^{2}=1$, the period of the signal $T$ being equal to 2 samples.

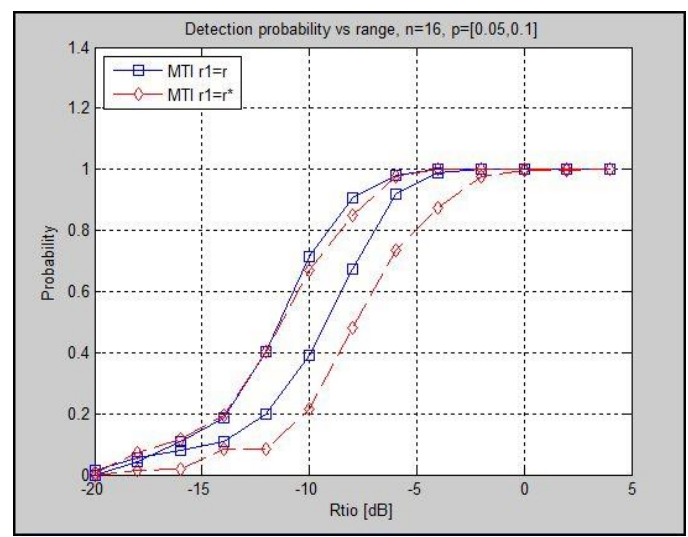

Fig. 6. Detection characteristics. Adaptive robust detector (the red curve) and non adaptive robust detector (the blue curve) for $p=0.03$ (the two upper curves) and $p=0.1$ (the two lower curves). The losses in the threshold signal at 0.9 are from 1 up to $2.5 \mathrm{~dB}$

These plots show that the losses due to adaptation are small and the adaptive algorithm that uses robust estimations of the correlation characteristics of noise is very efficient.

\section{Conclusion}

The pulse interference influences the characteristics of the classical algorithm of detecting moving targets (11), named MTI. The robust algorithm suggested in the paper is efficient under condition of pulse interference action. The gain is about $5 \mathrm{~dB}$. The proposed approach allows synthesis of new robust adaptive algorithms for signal detection in a difficult interference situation under the influence of correlated non-Gaussian impulsive noise. The results are important for numerous radar applications including a moving target detection on the sea background. 


\section{References}

1. Korniljev, E. A., I. G. Prokopenko, V. M. Chuprin. Ustoychivyje algoritmy v Avtomatyzirovannyh Sistemah Obrabotki Informatsyi. Kyiv, Tehnika, 1989 (in Russian).

2. Hu b e r, P. J. Robust Statistics. J. New York, Chichester-Brisbane, Toronto, Willey and Sons, 1981.

3. S a libia n-B arrera, M., S. V an A e lst. Robust Model Selection Using Fast and Robust Bootstrap. - Computational Statistics \& Data Analysis, Vol. 52, 2008, No 12, pp. 5121-5135.

4. M a r o n n a, R. A., R. D. M a r t i n, V. J. Y o h a i. Robust Statistics. Wiley, 2006. 